\title{
Structure, Water Permeability and Metabolism of Tilia miqueliana seeds during imbibition and dormancy release process
}

\section{YUWU}

Nanjing Forestry University https://orcid.org/0000-0002-4253-118X

Bao Shen ( $1484607714 @ q q . c o m$ )

https://orcid.org/0000-0002-2588-6296

\section{Research article}

Keywords: control seed, $\mathrm{H} 2 \mathrm{SO} 4$ treated seed, structure, water permeability, metabolism, imbibition, dormancy release

Posted Date: January 21st, 2020

DOI: https://doi.org/10.21203/rs.2.21487/v1

License: (c) (i) This work is licensed under a Creative Commons Attribution 4.0 International License.

Read Full License 


\section{Abstract}

The mechanical barrier and low permeability of seed coat and endosperm may be the main obstacle to the imbibition and dormancy release of Tilia miqueliana seeds. MRI, SEM, TEM and PSD were used to study the structure, water permeability and metabolism of control seeds and $\mathrm{H} 2 \mathrm{SO} 4$ treated seeds during imbibition and dormancy release. The results showed that $\mathrm{H} 2 \mathrm{SO} 4$ treatment greatly improved the permeability of seed coat and endosperm, accelerated the metabolism of high electron density substances in endosperm cells, and shortened the process of seed dormancy release and germination. At the same time, protoplasts that eventually developed into vascular bundles were surprisingly found in cotyledons and radicles.

\section{Background}

Tilia miqueliana tree, belonged to Tilia L.Tiliaceae, one of the unique linden tree species endemic to China. There are only 2,000 wild trees currently, which are endangered and rare species protected by the state. The wild population resources are few and in the period of growth decline(Yao, etc.,2018). The seeds have a long dormancy period and a low germination rate, resulting in poor natural reproduction and on the verge of extinction (Baskin, etc.,2004). Based on the study of seed structure characteristics of Tilia miqueliana seeds, the release of dormancy process was accelerated and the germination cycle was shortened by $\mathrm{H} 2 \mathrm{SO} 4$ treatment, which will provide theoretical basis for expanding seedling production in the future (Zhang, etc.,2010; Leubner-Metzger, etc.,2003).

\section{Results}

\subsection{Structural of the seed}

The Tilia miqueliana seed is approximately oval, with a micropyle at the tip point, a hilum at the end point, a dark brown seed coat, a raised back and a flat abdomen. The seed coat is thin, hard, and the surface is densely waxed. The endosperm is milky white, and the embryo is pale yellow. Two cotyledons are folded in the endosperm, and the hypocotyl and radicle are cylindrical (Fig. 2) (Creff, etc.,2015).

\subsection{Tissue observation in scanning electron microscope (SEM)}

It can be seen from the SEM image of the cross section that the seed coat was divided into three layers: outer layer, middle layer and inner layer (Fig. 3A1) (Sechet, etc.,2016). The hilum is in the shape of a circle and the cells are loosely arranged (Fig. 3A2), the inner surface of the micropyle is a point-like protrusion and the cells are closely arranged (Fig. 3A3). The outer layer was the stratum corneum (Fig. 3A4), the middle layer was composed of columnar cells, and the cells are arranged in parallel, relatively regular, and loosely arranged (Fig. 3A5). The inner layer was dense, the direction was perpendicular to the columnar cells of the middle layer (Fig. 3A6). The endosperm cells are mostly closely arranged in a gully shape with 
an irregular quadrilateral or pentagonal structure (Fig. 3B1,2). The cells in the longitudinal and transverse sections of the endosperm are highly dense and closely packed. (Fig. 3B3,4).

\subsection{Spatial distribution of water protons}

MR imaging was used to measure the spatial distribution of water in imbibition control seed and $\mathrm{H}_{2} \mathrm{SO}_{4}$ seed (Fig. 4A 4B). The images presented are limited to one 2D median or near-median slice in the axial planes and one superficial slice in the coronal plane. The slices were taken as a series of sections from single seed at a given time (Gruwel, M. L. etc.,2002 ; Gruwel, M. L. etc.,2004).

Figure 4A1 $(0 \mathrm{~h})$ shows that the initial water content in the control seed is mainly distributed in the hypocotyl. AfterA2(4h) of soaking, water enters radicle through micropyle. After A3(13 h), A4(20 h), $A 5(28 h), A 6(38 h), A 7(48 h)$ and $A 8(60 h)$ of imbibition, moisture enters the cotyledons from the hilum and continues to enter radicle and hypocotyl through micropyle. After soaking for A9(72 h), A10(82 h), $A 11(97 \mathrm{~h}), A 12(120 \mathrm{~h}), A 13(138 \mathrm{~h}), A 14(161 \mathrm{~h})$ and A15(185 h), the red water signal in the cotyledons, radicles and hypocotyls is more pronounced, and obvious red water signals begin to appear on both sides of the endosperm. After imbibition for A16(42d), A17(46d), A18(51d), A19(57d), A20(63d) and A21(71d), the red water signals on the cotyledons, radicles, hypocotyls, and outside endosperms are more apparent, but there is no water signal on the inside of the endosperm.

Figure 4B1 $(0 \mathrm{~h})$ shows that there was no obvious water signal in the $\mathrm{H}_{2} \mathrm{SO}_{4}$ treated seeds. After 3B2 $(2 \mathrm{~h}), 3 B 3(4 \mathrm{~h}), 3 B 4(6 \mathrm{~h}), 3 B 5(8 \mathrm{~h}), 3 B 6(14 \mathrm{~h}), 3 B 7 \mathrm{(23} \mathrm{h})$ and 3B8 $(30 \mathrm{~h})$ of soaking, water enters the radicle through the micropyle. After imbibition for B9 (38 h), B10 (48 h), B11 (58 h), B12 (70 h), B13(82 h), B14 (92 h), B15 (107 h), B16 (130 h), B17(148 h), B18 (171 h) and B19 (195 h), water enters cotyledons through the hilum, and penetrates into endosperm on both sides through micropyle and hilum. After soaking for B20(21d), B21(31d), B22(35d), B23(42d), B24(46d) and B25(51d), water enters into the whole seed coat and the inside of the endosperm, and the red water signal of the whole embryo is more pronounced. After B26(57d), B27(63d) and B28(71d) of soaking, the entire seed coat, endosperm, embryo cavity and embryo all showed strong red water signals.

The SNR data of control seeds during imbibition and cold stratification process (Fig. 5A). The imbibition phase I (0-185 h), the SNR value of hilum showed three times ups and downs, and the amplitude of second rise and fall was larger; the SNR value of micropyle showed three times ups and downs, then continue to rise; the SNR value of seed coat experienced three sharp rises and falls, and then continued to rise; the SNR value of seed coat experienced two times small amplitude of rises and falls, and then continued to rise. The imbibition phase II (185 h-744 h), the SNR values of hilum, micropyle, seed coat and endosperm showed continuous decline to saturation. The saturation stage (744 h- $1512 \mathrm{~h}$ ), the SNR value of hilum showed falling after rising, and almost flat after saturation; the SNR value of micropyle showed rising after rising and falling, and slowly decrease after saturation; the SNR value of seed coat showed falling after rising, and slowly decrease after saturation ,the SNR value of seed coat showed two times sharp rises and falls, and then rise rapidly after saturation (1512 h-1704 h). 
The SNR data of $\mathrm{H}_{2} \mathrm{SO}_{4}$ treated seeds during imbibition and cold stratification process (Fig. 5B). The imbibition phase I ( $0-185 \mathrm{~h})$, the SNR value of hilum rises and falls, then rises again; the SNR value of micropyle rose again after two times of rises and falls, and the range of first time rises and falls was sharply obvious; the SNR value of seed coat has gone through three times of sharp rises and falls; the SNR value of endosperm showed three smaller amplitudes rises and falls. The imbibition phase II (185 h$744 \mathrm{~h}$ ), the SNR value of hilum and micropyle continued to decrease to saturation; the SNR value of seed coat falls to saturation after sharply falls and rises; the SNR value of endosperm smoothly rises and falls to saturation. The saturation stage (744 h- $1104 \mathrm{~h}$ ), the SNR values of hilum, micropyle, seed coat and endosperm showed continues to rise and then decline. The germination phase (1104 h- $1704 \mathrm{~h}$ ), the SNR value of hilum and endosperm rises slowly; the SNR value of seed coat and micropyle showed the same trend, which increased rapidly to $1224 \mathrm{~h}$ (51d), then decreased rapidly to $1512 \mathrm{~h}$ (63d), and then increased. We found that the SNR value of micropyle and seed coat was the highest at $1224 \mathrm{~h}(51 \mathrm{~d})$, which was probably the key point of seed dormancy release.

\subsection{Tissue observation in PSD}

We using PSD to detect the changes of morphological properties of endosperm cells in control seed and $\mathrm{H}_{2} \mathrm{SO}_{4}$ treated seeds. The elongated thick-walled cells are closely arranged in the elongation zone of the cotyledons (Fig. 6A1). The long strips of tetragonal parenchyma cells are closely arranged in the procambium of cotyledon (Fig. 6A2). The bud tip of the germ consists of irregular thick-walled cells

(Fig. 6A3). The endosperm consists of a stack of tetragonal parenchyma cells (Fig. 6A4). The elongated thick-walled cells are closely arranged in the elongation of the radicles (Fig. 6A5). The root end of the radicles is composed of irregular thick-walled cells (Fig. 6A6). The long strips of tetragonal parenchyma cells are closely arranged in the cell division area (Fig. 6A7).

The procambium of cotyledon and the elongation of the radicles will eventually develop into vascular bundles. The morphological properties of endosperm cells in control seed have no obvious change in cold stratification (Fig. 6B). After 35 days of cold stratification, the morphological properties of endosperm cells in $\mathrm{H}_{2} \mathrm{SO}_{4}$ treated seeds changes from an elongated state to a small polygonal shape, and the arrangement becomes tight (Fig. 6C5). After 55 days of cold stratification, the morphological properties of endosperm cells become polygonal, containing small round particles and closely packed (Fig. 6C9)(Sechet and Frey et al., 2016). After 60 days of cold stratification, the red granules in the outer parenchyma cells of the endosperm have been degraded and the radicle has broken through the endosperm cap (Fig. 6C10). It can be seen that $\mathrm{H}_{2} \mathrm{SO}_{4}$ treatment accelerated the degradation of endosperm contents and shortened the time of seed dormancy release.

\subsection{Tissue observation in TEM}

The endosperm cells of control seed contained a large number of circular, elliptical, and polygonal high electron dense substances, which are surrounded by lipids (Fig. 7A1). After 10 days of cold stratification, the endosperm cells still contained more lipid particles and a large amount of high electron dense 
substances (Fig. 7A2). On the 20th days of cold stratification, the lipids began to decompose, but the high electron dense substance did not change (Fig. 7A3). On the 30th days of cold stratification, the lipid in the endosperm cell continued to decomposed and a large amount of high electron density substance still remained (Fig. 7A4). On the 40th day of cold stratification, the lipids gradually ablated and decomposed into small vacuoles, and there is no change in the high electron density substance (Fig. 7A5). On the 50th day of cold stratification, the lipids gradually ablated and decomposed into big vacuoles, but the high electron dense substance showed no change (Fig. 7A6). On the 55th day of cold stratification, the lipids gradually ablated and decomposed into vacuoles, and a few irregular shaped high electron dense materials began to degrade (Fig. 7A7)(Bewley, 1997). On the 60th day of cold stratification, most of the lipid droplets have broken down, and the round and elliptical high electron dense matter begins to degrade in small portion (Fig. 7A8). In conclusion, during the whole cold stratification process, the high electron dense substance in the cells of the control seed degraded very slowly, but the lipids were decomposed almost completely.

Part of the round high-electron dense substance in the endosperm cells after soaking for 15 min in $\mathrm{H}_{2} \mathrm{SO}_{4}$ showed obvious holes and cracks, and containing a lot of lipid droplets (Fig. 7B1). After 10 days of cold stratification, the pores and cracks of the high electron dense substance become larger and more pronounced, and the irregularly high electron dense substance begins to degrade, and the amount of lipid droplets are still existed (Fig. 7B2). After 20 days and 30 days of cold stratification, the irregularly high electron dense substances continue to degrade (Fig. 7B3,4). After 40 days of cold stratification, the irregular high-electron dense substances degraded significantly, and round and elliptical high-electron dense substances begin to degrade initially (Fig. 7B5). After 50 days of cold stratification, the round high electron dense substance is obviously degraded (Fig. 7B6). After 55 days of cold stratification, the round high electron dense substance is highly degraded (Fig. 7B7). After 60 days of cold stratification, the high electron density substance degraded into white vacuoles which contained the residual black fragments that are not thoroughly degraded, and still contained a large amount of lipid droplets (Fig. 7B8). The most obvious change in the cold stratification process of $\mathrm{H}_{2} \mathrm{SO}_{4}$ treated seeds is that there are obvious holes and cracks in the high electron density substance in the cells, and the degradation and ablation speed are accelerated(Leubner-Metzger, 2003). Obvious pores and cracks undoubtedly greatly contribute to the improvement of endosperm permeability (Finch Savage, etc.,2006).

\section{Discussion}

\subsection{Effects of structure and permeability characteristics of seed coat and endosperm on seed dormancy}

The dense array of seed coat and endosperm cells creates mechanical barriers and poor permeability characteristics for the seeds. The most obvious change in the $\mathrm{H}_{2} \mathrm{SO}_{4}$ treated seeds is that there are obvious holes and cracks on the surface of the high electron density substance cells, it undoubtedly contributed to the improvement of endosperm permeability. The water inlet speed was accelerated, and eventually penetrates into the seed cavity, embryo and endosperm, and reached the requirement for 


\subsection{Effect of $\mathrm{H}_{2} \mathrm{SO}_{4}$ treatment on the process of seed imbibition and dormancy release}

In the process of cold stratification, the morphological properties of endosperm cells in control seed have no obvious change, on the contrary, it has an obvious change in $\mathrm{H}_{2} \mathrm{SO}_{4}$ treated seeds. The endosperm cells changes from an elongated state to a small polygonal shape, and the arrangement becomes tight, then the endosperm cells become polygonal, containing small round particles and closely packed, At the end, the red granules in the outer parenchyma cells of the endosperm have been degraded and the radicle has broken through the endosperm cap. At the same time, the high electron dense substance in the cells of the control seed degraded very slowly, but the lipids were decomposed almost completely. However, in the endosperm cells treated with $\mathrm{H}_{2} \mathrm{SO}_{4}$, almost all the high electron dense substances in various shapes were degraded, but lipid droplets still existed. $\mathrm{H}_{2} \mathrm{SO}_{4}$ treatment accelerated the degradation of endosperm contents and shortened the time of seed dormancy release.

\section{Conclusion}

In conclusion, it is clear that $\mathrm{H}_{2} \mathrm{SO}_{4}$ plays an important role in eliminating the mechanical barrier and promoting the permeability of seed coat and endosperm (Schopfer, etc.,1984; Resendiz-Vazquez, etc.,2017). We observed the cells of the seed coat and endosperm are highly dense and closely packed by SEM. At the same time, we detected that water entered cotyledon and radicle through micropyle and hilum by MRI. On the 51d, there was a strong water signal in the endosperm and all the seed coats, and the SNR value of micropyle and seed coat was the highest, which was probably the Key point of seed germination. Through PSD detection, we found that the unique feature of the Tilia miqueliana seed is that the procambium of cotyledon and the elongation of the radicles will eventually develop into vascular

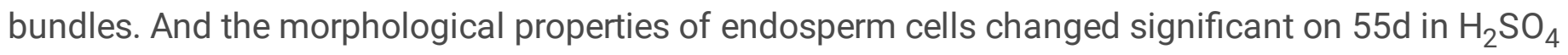
treated seeds. The TEM examination also showed that the round high electron dense substance was highly degraded at $55 \mathrm{~d}$ in $\mathrm{H}_{2} \mathrm{SO}_{4}$ treated seeds. All the evidences showed that the permeability of the seed coat and endosperm was improved after soaking in $\mathrm{H}_{2} \mathrm{SO}_{4}$, which accelerated the structural change of endosperm cells and metabolism of cell contents, and the $50 \mathrm{~d}-55 \mathrm{~d}$ is the key period of seed dormancy release.

\section{Methods}

\subsection{Seeds and seeds treatment}


Research permission on Tilia miqueliana tree has been obtained from Jiangsu Wildlife Protection Station. With the permission of the Huang zangyu National Forest Park Management Office, the seeds used the experiment were harvest in November 2018, Huang zangyu National Forest Park, Anhui Province, China. The Southern Forest Seed Inspection Center of the State Forestry and Grassland Bureau has determined the quality and viability of the seeds. First, plump fruits were selected by water, and the husks are removed manually to take out the seeds. Then, full seeds were selected by water, dried in the room, and stored in a refrigerator at 4 ' for cold storage. The moisture content of mature seeds was $9.74 \%$. The $100-$ grain weight of the seeds was $22.54 \mathrm{~g}$.

The experiment was divided into control seeds and $\mathrm{H}_{2} \mathrm{SO}_{4}$ treated seeds. The control seeds were studied without any additional treatment, and soaked in running water at $17^{\circ} \mathrm{C}$ for $185 \mathrm{~h}$. The $\mathrm{H}_{2} \mathrm{SO}_{4}$ treated seeds were soaked in $98 \% \mathrm{H}_{2} \mathrm{SO}_{4}$ for $15 \mathrm{~min}$, then soaked in running water at $17^{\circ} \mathrm{C}$ for $195 \mathrm{~h}$.

Subsequently the control seeds and $\mathrm{H}_{2} \mathrm{SO}_{4}$ treated seeds were transferred to wet sand for 80 days of cold stratification to observed germination.

The voucher specimen of this material has been deposited in Southern Forest Seed Inspection Center of the State Forestry and Grassland Bureau, and the deposition number was 2019-NJD-11.

\subsection{Preparation of tissues for scanning electron microscopy (SEM)}

The material morphology and cross section structure of seed coat and endosperm were subtle analyzed by SEM. Two control seeds and two $\mathrm{H}_{2} \mathrm{SO}_{4}$ treated seeds of 0 day were selected for treatment, and dried in an oven of $40^{\circ}$ for 4 hours. The seed coat and endosperm were cut into 2 square millimeters of transverse sections, fixed on the sample table with double-sided tape, then sprayed a thin gold film on the surface, and observed under scanning electron microscope (FEI, USA).

\section{$5.3^{1} \mathrm{H}$ MRI}

All magnetic resonance experiments were performed on 7.0 T MRI scanner (PharmaScan, Bruker Biospin $\mathrm{GmbH}$, Germany) using a quadrature surface RF coil(Terskikh and Feurtado et al., 2005). Every single seed was fixed on the $25 \times 75 \mathrm{~mm}$ microscope slides with $A B$ glue, and put into the $45 \mathrm{ml}$ centrifuge tube filled with water, then placed into a $38 \mathrm{~mm}$ volume coil. Precautions were taken to ensure that no air bubbles were trapped to avoid ambiguous interpretation of the MR images. The exact location of the seed in the coil and its orientation were determined with a few fast scout MRI scans and the axial section was selected for further study. Typically, the first in a series of in situ MRI experiments was started immediately after the first contact of the seed with water (Terskikh, etc., 2005). Subsequent swelling experiments were performed at different intervals for 185-195 hours, and germination experiments were performed at different time intervals for 80 days. To study imbibition of the seeds in situ we used water in imbibition phase and wet sand in germination process as an artificial soil substrate respectively. ${ }^{1} \mathrm{H}$ MRI experiments were performed at a resonance frequency of $300.337 \mathrm{MHz}$ at $22 \pm 1 \mathrm{C}$. Proton intensity MR images were recorded with a RARE-pd. Anatomical images were acquired with a turbo-rapid acquisition 
relaxation enhancement (RARE) PD-weighted sequence (repetition time (TR)/echo time (TE) = $1033 / 10 \mathrm{~ms}$, slices $=27$, field of view $(F O V)=2.8 \times 2.8 \mathrm{~cm}$, number of averages $=15$, matrix $=256 \times 256$, slice thickness $/ \mathrm{gap}=0.3 / 0 \mathrm{~mm}$, flip angle $=180^{\circ}$ ), scan time $=15 \mathrm{~min}$, pixel resolution $=109 \mathrm{um}$.

\subsection{Preparation of tissues for transmission electron microscopy (TEM)}

The changes of endosperm cells metabolism in seeds cold stratification process at $0 \mathrm{~d}, 10 \mathrm{~d}, 20 \mathrm{~d}, 30 \mathrm{~d}$, 35d, 40d, 45d, 50d, 55d and 60d were examined by TEM. On each sampling point, the tissues taken from the endosperm of one control seed and $\mathrm{H}_{2} \mathrm{SO}_{4}$ seed were cut into 1 cubic millimeter of block, and immediately fixed in electron microscopic fixative (Servicebio, Wuhan, China) for 2 hours, then transferred to fixed in $1 \%$ OsO4 for 5 hours, then dehydration through the ethanol series and finally embedded in acetone blocks. The acetone blocks were sliced on a microtome with a glass knife to a thickness of $60 \mathrm{~nm}$ and stained in $5 \%$ uranyl acetate and $0.5 \%$ lead citrate, then observed under the transmission electron microscope HT7700 (Hitachi, Tokyo, Japan).

\subsection{Preparation of tissues for paraffin section detection (PSD)}

The changes of endosperm cell structure and metabolism in seeds cold stratification process at $0 \mathrm{~d}, 10 \mathrm{~d}$, 20d, 30d, 35d, 40d, 45d, 50d, 55d and 60d were examined by PSD. On each sampling point, two control seeds and two $\mathrm{H}_{2} \mathrm{SO}_{4}$ treated seeds were selected to cut longitudinal dissection into two halves and fixed in $70 \%$ FAA fixative (Servicebio, Wuhan, China) for $24 \mathrm{~h}$, then dehydrated through the ethanol series and finally embedded in paraplast. The tissues taken from the endosperm were cut using glass knives on an RM2016 (laika, Shanghai, China) ultramicrotome to a thickness of $3 \mu \mathrm{m}$, and stained with safranine and fast green (Jensen 1962). And observed under a light microscope (Nikon, Tokyo, Japan).

\section{Abbreviations Used}

MRI, magnetic resonance imaging; SNR, signal-to-noise ratio; SEM, scanning electron microscopy; TEM, transmission electron microscopy; PSD, paraffin section detection.

\section{Declarations}

\section{Ethics approval and consent to participate}

\section{Legal and Licences statement}

We strictly obeyed comply with the Convention on the Trade in Endangered Species of Wild Fauna and Flora, and also accordance with Law of the People's Republic of China on Wildlife Protection. Research permission on Tilia miqueliana tree has been obtained from Jiangsu Wildlife Protection Station. The collection of experimental seeds was approved by the Management Office of Huang zangyu National Forest Park in Anhui Province, China. 


\section{Consent for publication}

Not applicable

\section{Availability of data and materials}

All data generated or analysed during this study are included in this published article.

\section{Competing interests}

The authors declare that they have no competing interests.

\section{Funding}

This work was supported by the Priority Academic Program Development of Jiangsu Higher Education Institutions (PAPD), the PAPD provides the full cost of the experiment.

\section{Authors' contributions}

YBS conceived the original screening and research plans; YW performed the experiments using the MRI, SEM, TEM and PSD methods; YW designed the experiments and analyzed the data; YW conceived the project and wrote the article with contributions of all the authors. YW agrees to serve as the author responsible for contact and ensures communication. All authors have read and approved the manuscript.

\section{Acknowledgements}

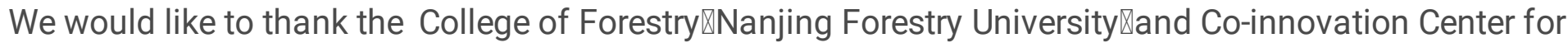
Sustainable Forestry in Southern China『Southern Tree Inspection Center National Forestry Administration.

\section{Authors' information (optional)}

Yu Wu, ${ }^{\dagger 1}$ Yong bao Shen, ${ }^{*},+\neq$

${ }^{\dagger}$ College of Forestry $\llbracket$ Nanjing Forestry University $₫$ Nanjing $\bigotimes$ Jiangsu province, China

${ }^{\ddagger}$ Co-innovation Center for Sustainable Forestry in Southern China $₫$ Southern Tree Inspection Center National Forestry Administration

Corresponding Author Yong bao Shen ㅌE-mail: 1484607714@qq.com

Author E-mail Yu Wu ${ }^{1}$ E-mail: 550868021@ qq.com

\section{References}


1. Baskin, J. M. and C. C. Baskin (2004). "A classification system for seed dormancy." Seed science research 14 (1): 1-16.

2. Bewley, J. D. (1997). "Breaking down the walls-a role for endo- $\beta$-mannanase in release from seed dormancy?" Trends in Plant Science 2 (12): 464-469.

3. Creff, A. and L. Brocard, et al. (2015). "A mechanically sensitive cell layer regulates the physical properties of the Arabidopsis seed coat." Nature Communications 6: 6382.

4. Finch Savage, W. E. and G. Leubner Metzger (2006). "Seed dormancy and the control of germination." New phytologist 171 (3): 501-523.

5. Gruwel, M. L. and P. Latta, et al. (2004). "Magnetic resonance imaging of seeds by use of single point acquisition." Journal of agricultural and food chemistry 52 (16): 4979-4983.

6. Gruwel, M. L. and X. S. Yin, et al. (2002). "Barley viability during storage: use of magnetic resonance as a potential tool to study viability loss." Journal of agricultural and food chemistry 50 (4): 667-676.

7. Leubner-Metzger, G. (2003). "Functions and regulation of $\beta$-1, 3-glucanases during seed germination, dormancy release and after-ripening." Seed Science Research 13 (1): 17-34.

8. Manz, B. and K. Müller, et al. (2005). "Water uptake and distribution in germinating tobacco seeds investigated in vivo by nuclear magnetic resonance imaging." Plant physiology 138 (3): 1538-1551.

9. Resendiz-Vazquez, J. A. and J. A. Ulloa, et al. (2017). "Effect of high-intensity ultrasound on the technofunctional properties and structure of jackfruit (Artocarpus heterophyllus) seed protein isolate." Ultrasonics sonochemistry 37: 436-444.

10. Schopfer, P. and C. Plachy (1984). "Control of seed germination by abscisic acid: II. Effect on embryo water uptake in Brassica napus L." Plant Physiology 76 (1): 155-160.

11. Sechet, J. and A. Frey, et al. (2016). "Xyloglucan metabolism differentially impacts the cell wall characteristics of the endosperm and embryo during Arabidopsis seed germination." Plant physiology 170 (3): 1367-1380.

12. Terskikh, V. V. and J. A. Feurtado, et al. (2005). "Water uptake and oil distribution during imbibition of seeds of western white pine (Pinus monticola Dougl. ex D. Don) monitored in vivo using magnetic resonance imaging." Planta 221 (1): 17-27.

13. Yao, W. and Y. Shen (2018). "Effects of gibberellic acid and magnetically treated water on physiological characteristics of Tilia miqueliana seeds." Canadian Journal of Forest Research 48 (5): 554-558.

14. Zhang, J. and B. R. Nallamilli, et al. (2010). "OsMADS6 plays an essential role in endosperm nutrient accumulation and is subject to epigenetic regulation in rice (Oryza sativa)." The Plant Journal 64 (4): 604-617.

\section{Figures}




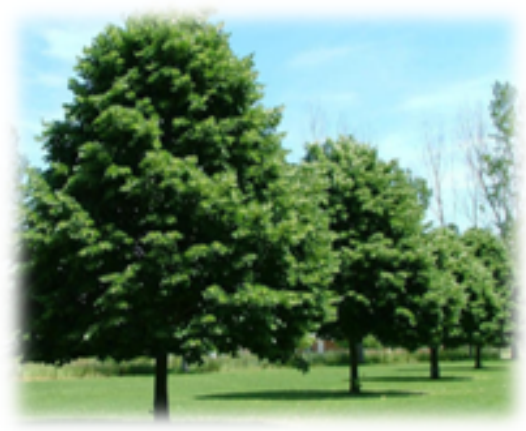

Trees

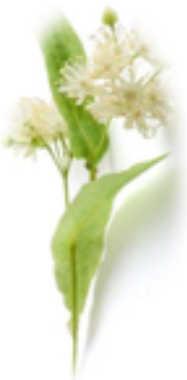

Leaflike bracts

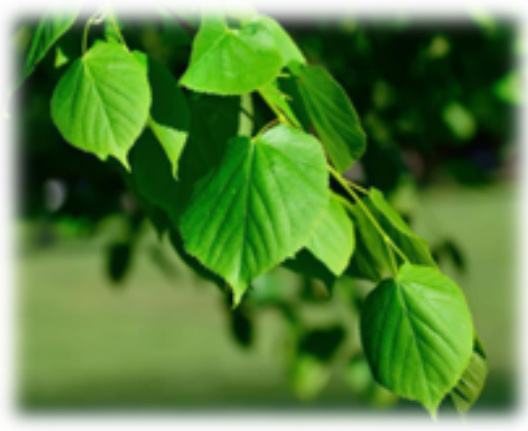

Leaves

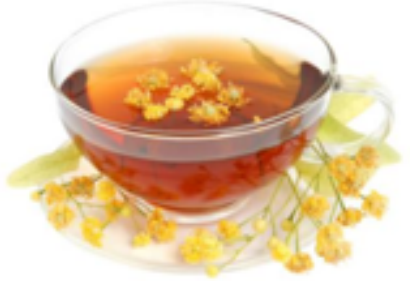

Linden tea

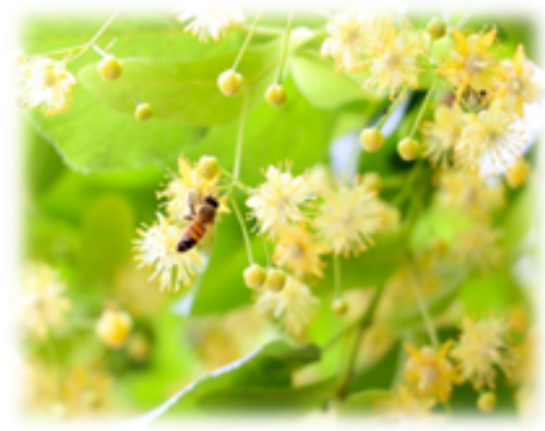

Flowers

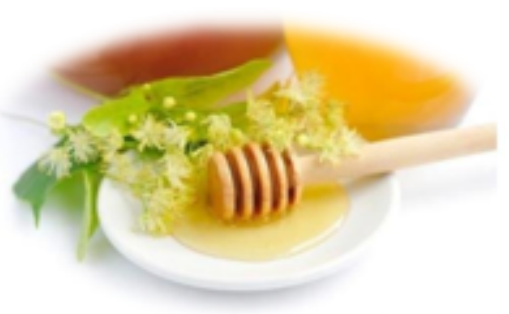

Linden honey

\section{Figure 1}

Digital photos of Tilia miqueliana trees, leaves, flowers, leaflike bracts and uses.

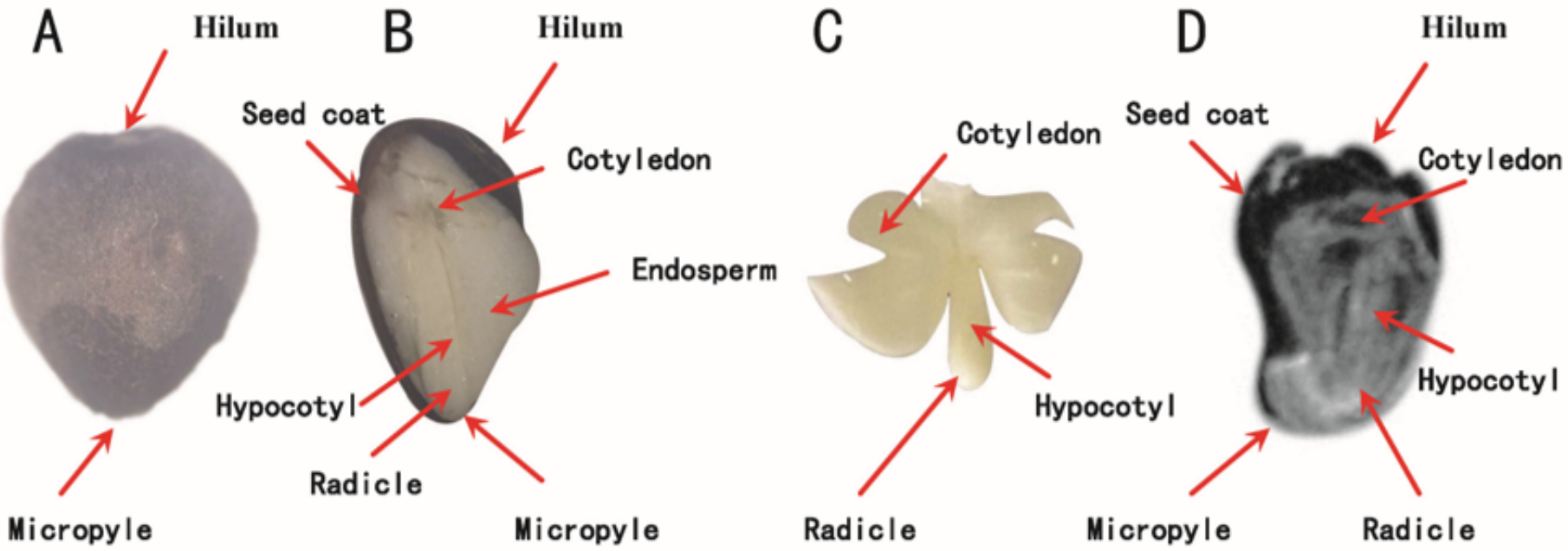

Figure 2

Anatomical structure of dry seeds 


\section{A Seed coat of control seeds}
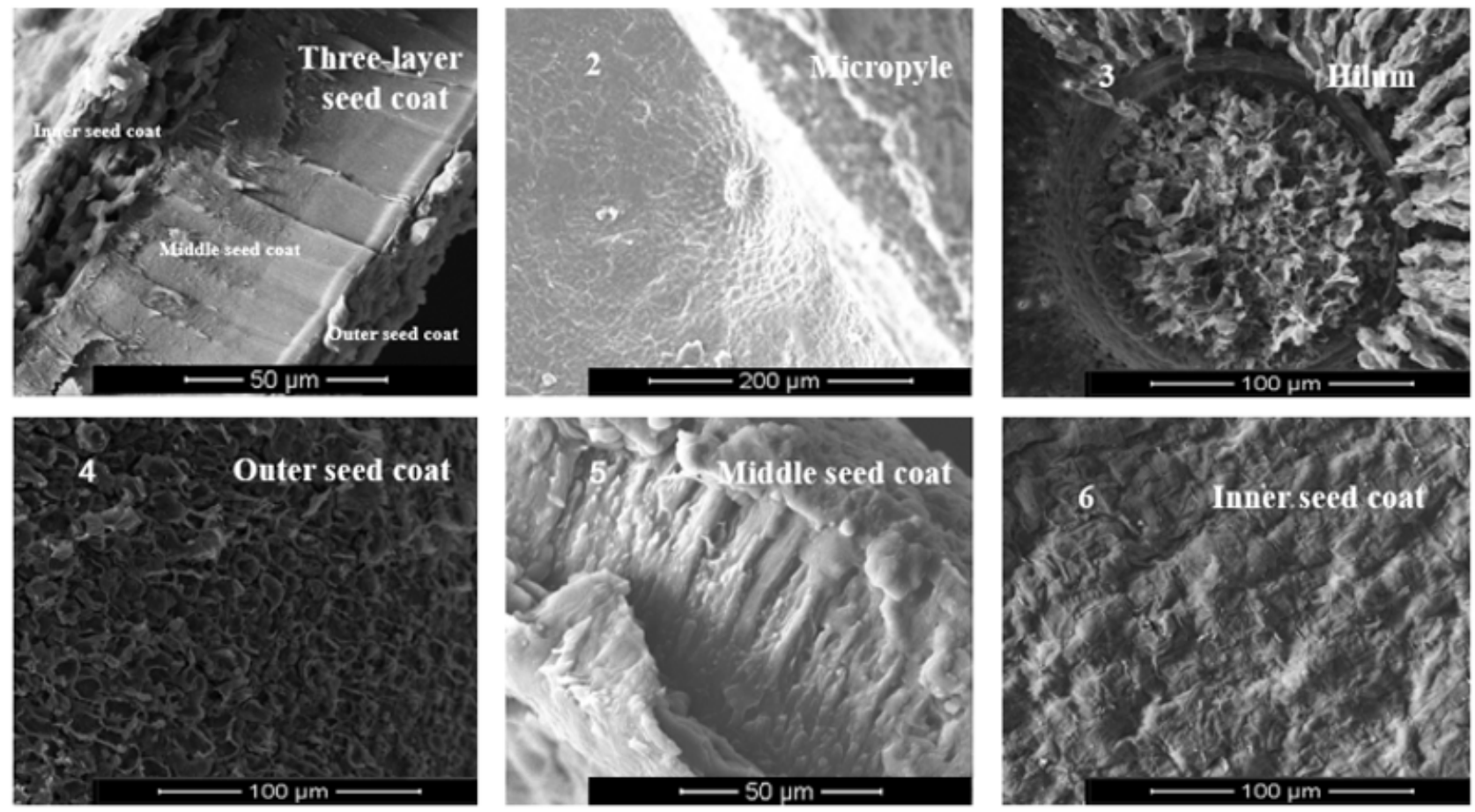

B Embryo of control seeds
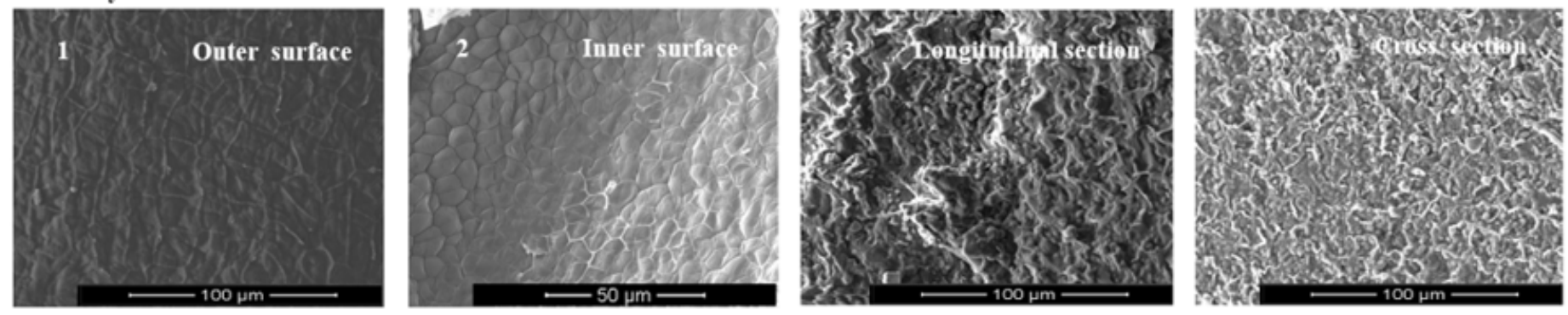

Figure 3

Scanning electron microscope of the dry seed coat and the endosperm 
A Control seed
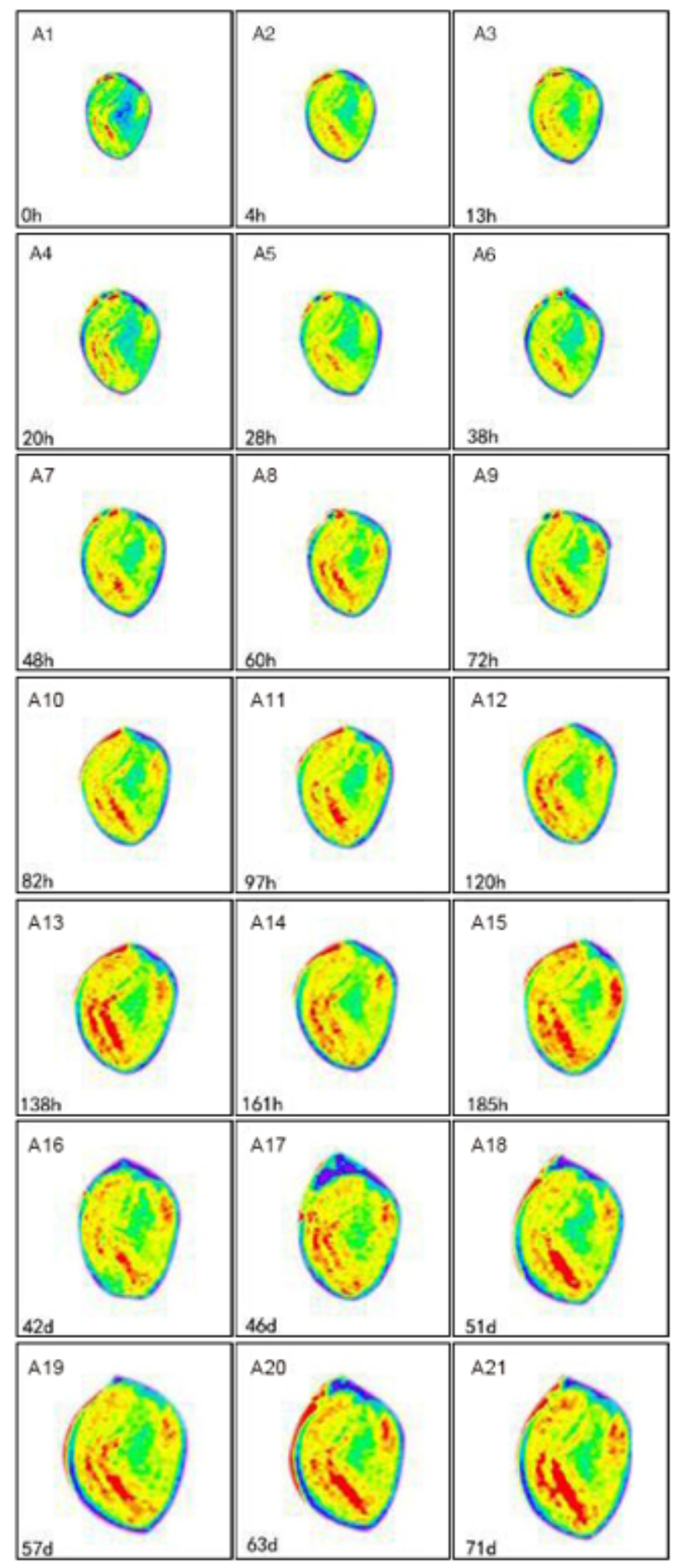

B $\mathrm{H}_{2} \mathrm{SO}_{4}$ treated seed

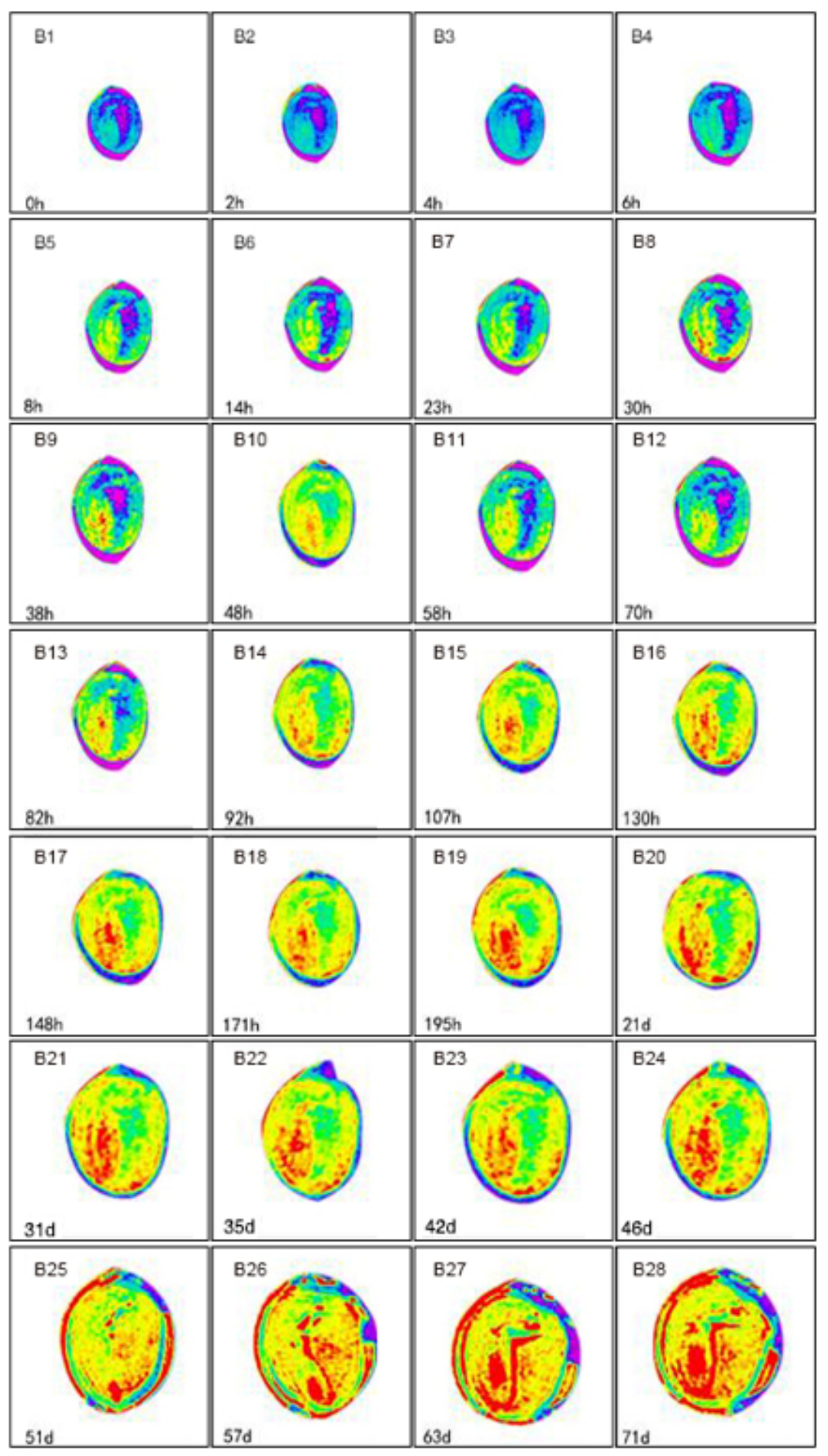

Figure 4

MR images of control seed and $\mathrm{H} 2 \mathrm{SO} 4$ treated seed 


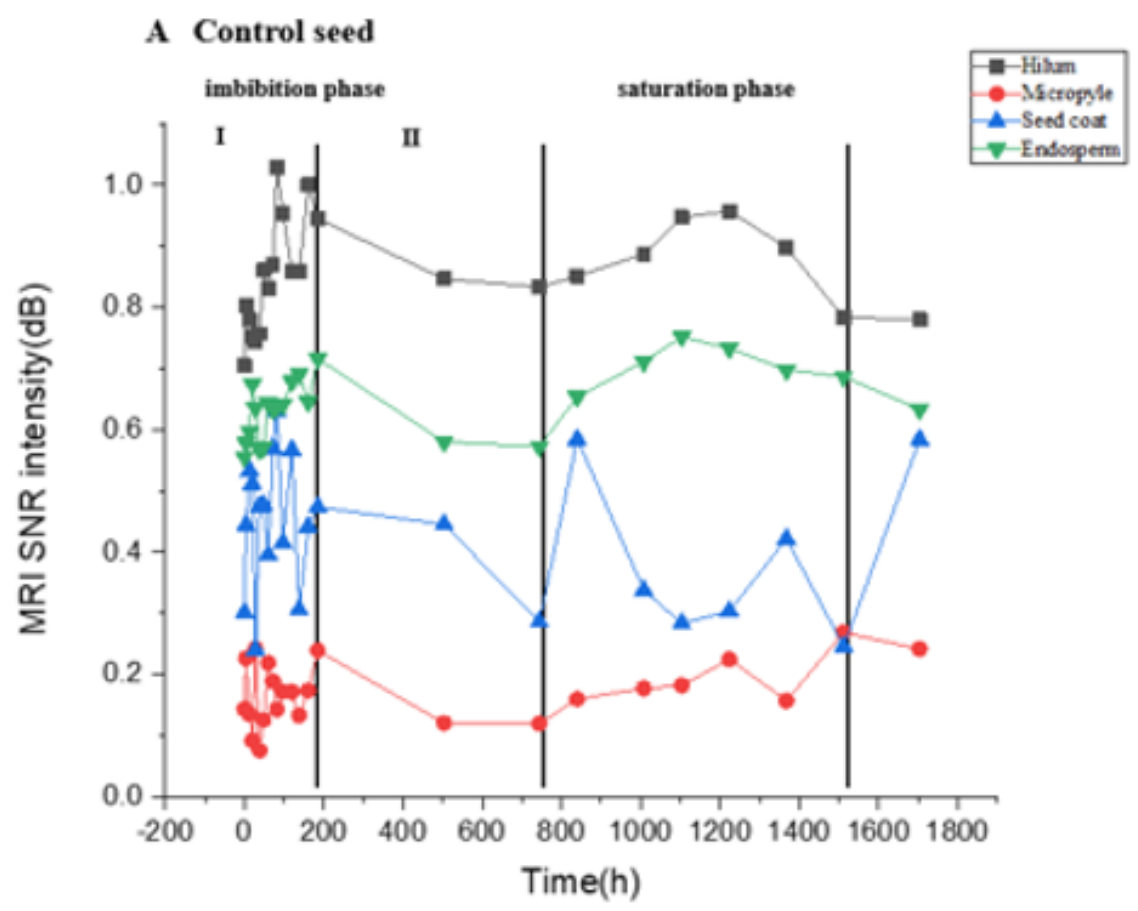

B $\mathrm{H}_{2} \mathrm{SO}_{4}$ treated seed

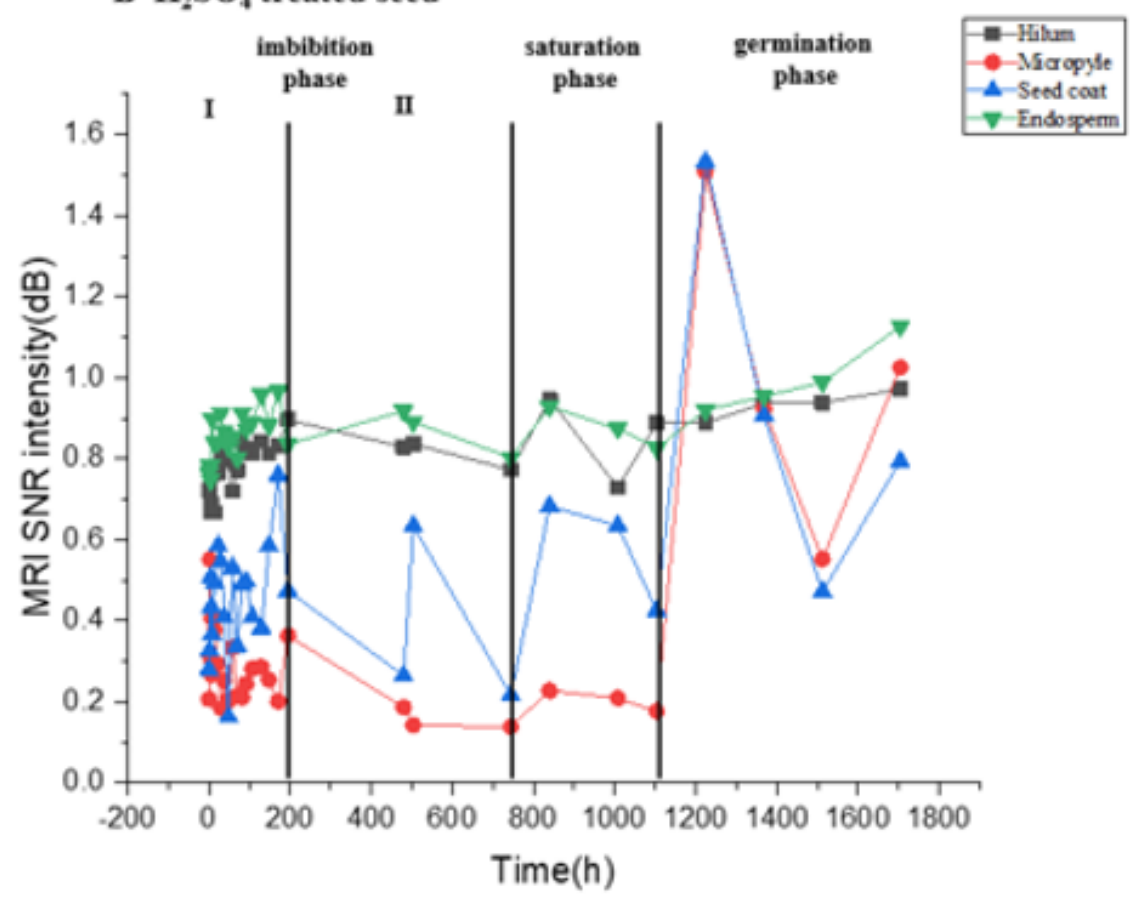

Figure 5

SNR data of control seed and $\mathrm{H} 2 \mathrm{SO} 4$ treated seed during imbibition and cold stratification process 

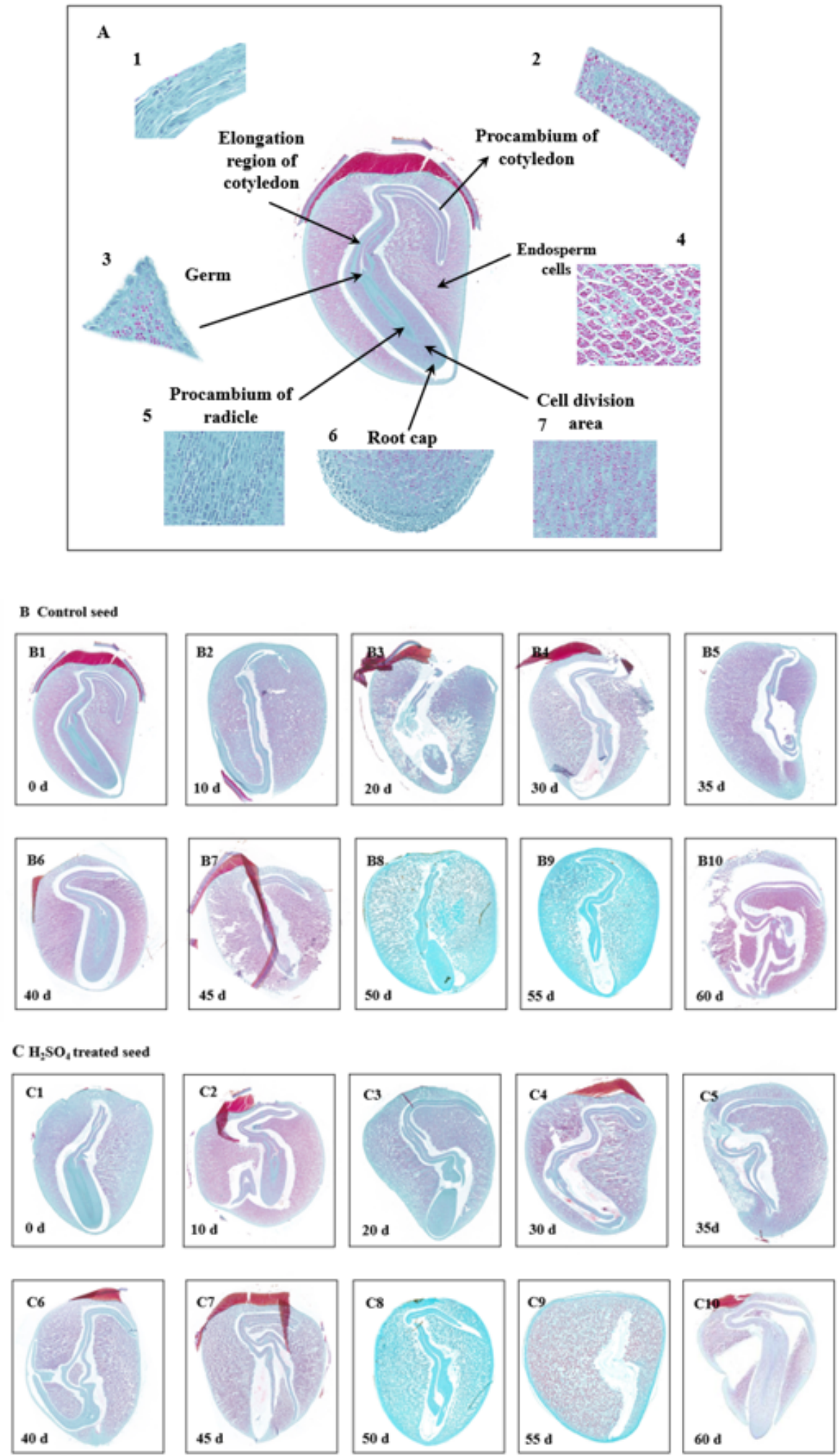

\section{Figure 6}

Red-green paraffın slice diagram of longitudinal section of control seed and H2SO4 treated seed 


\section{A Control seeds}
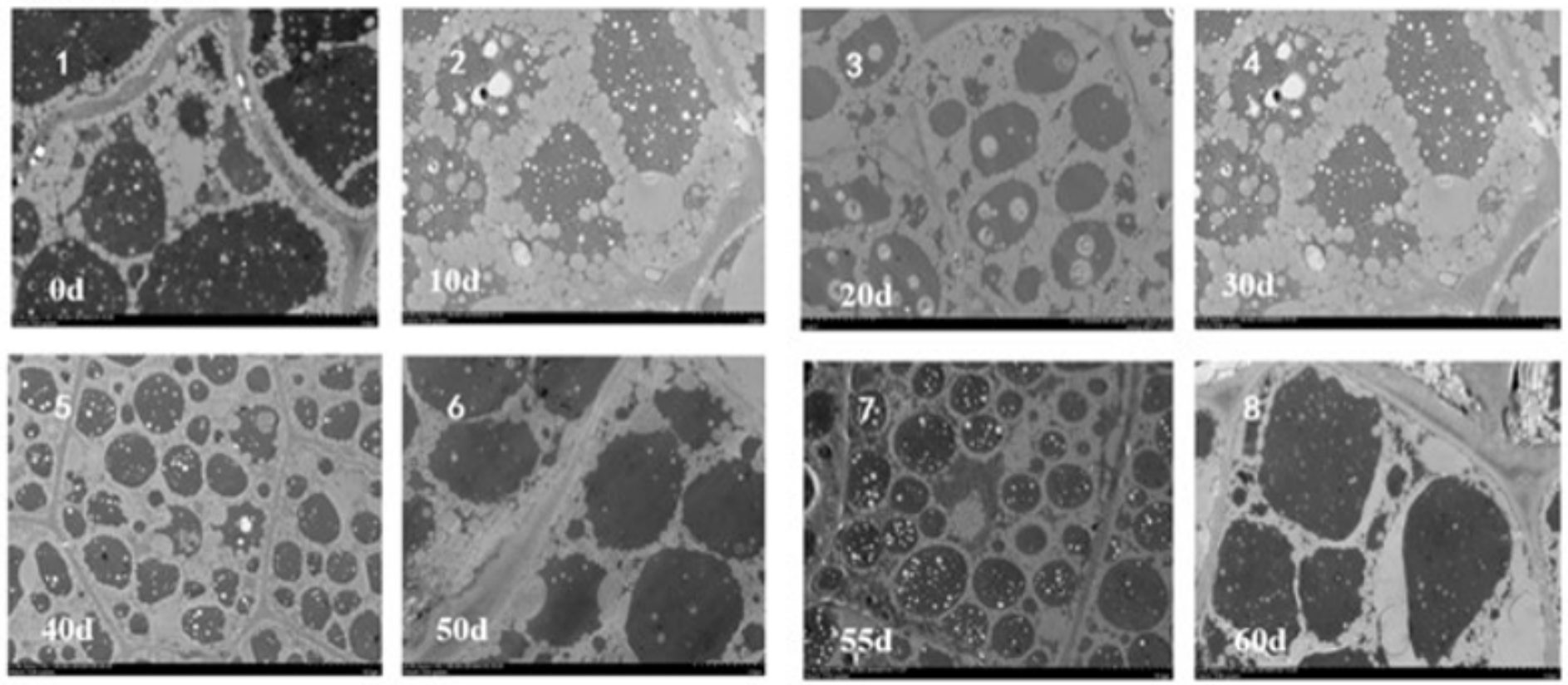

\section{B $\mathrm{H}_{2} \mathrm{SO}_{4}$ treated seeds}
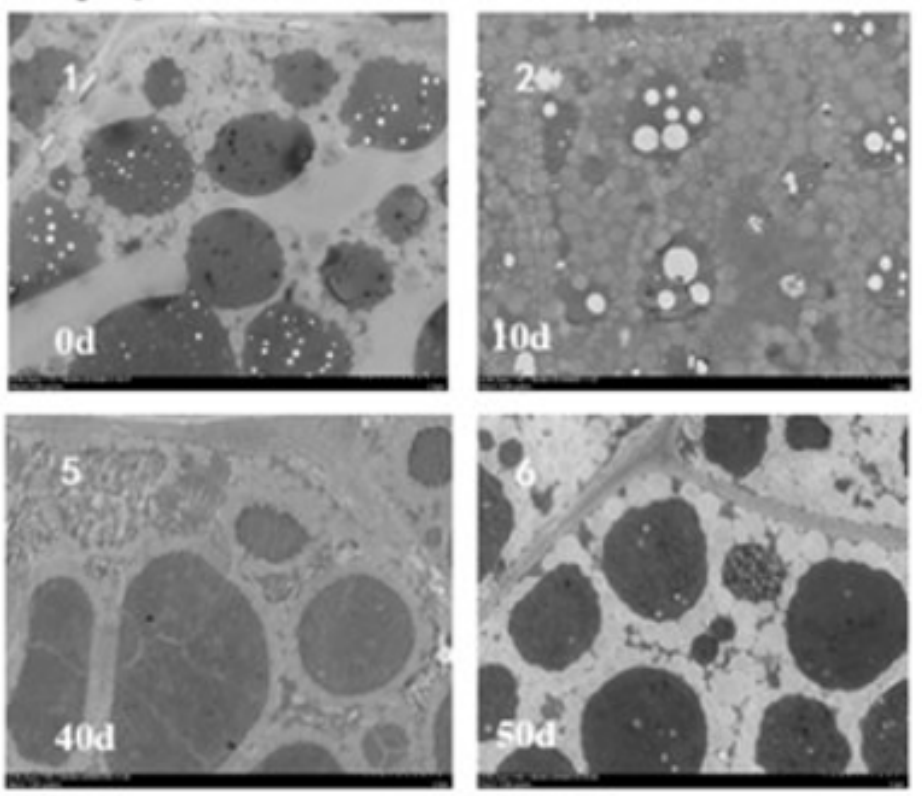
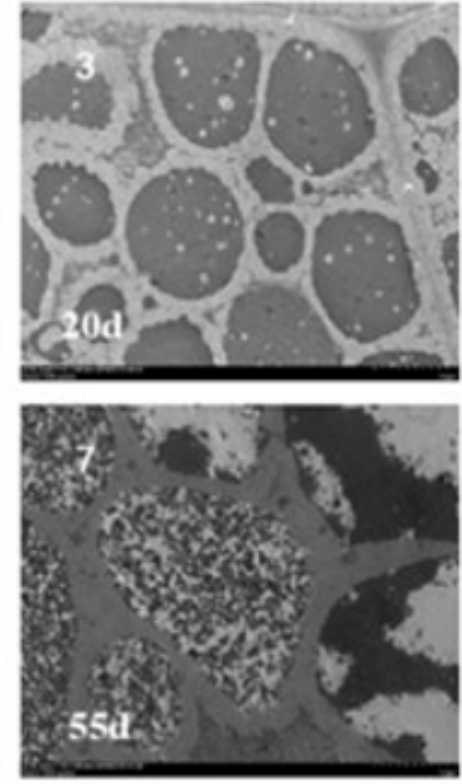
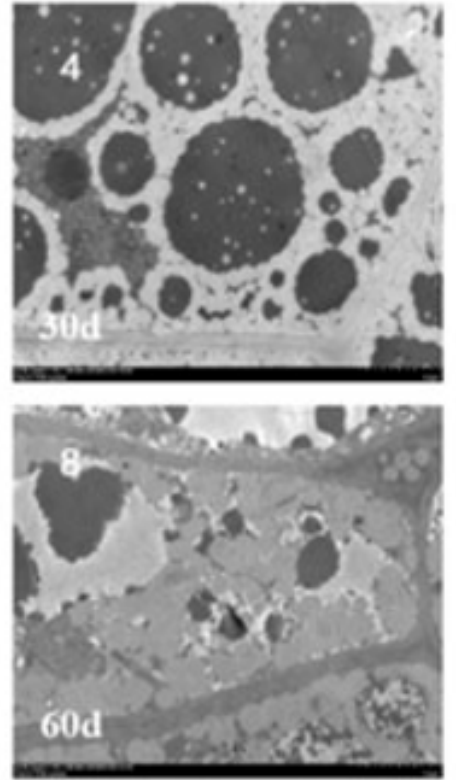

\section{Figure 7}

TEM images of endosperm cell contents of control seeds and $\mathrm{H} 2 \mathrm{SO} 4$ treated seeds 